\title{
A New Approach to Incorporate Uncertainty in Terrain Modeling
}

\author{
Jorge Santos ${ }^{1}$, Weldon A. Lodwick ${ }^{2}$ and Arnold Neumaier ${ }^{3}$ \\ ${ }^{1}$ Secção de Engenharia Geográfica, Departamento de Matemática, Fac. de \\ Ciências e Tecnologia, Universidade de Coimbra, Apartado 3008, 3001 - 454 \\ Coimbra, Portugal \\ jmfs@mat.uc.pt \\ ${ }^{2}$ Mathematics Department, University of Colorado at Denver, Campus Box 170, \\ P.O.Box 173364, Denver, Colorado USA \\ weldon.lodwick@cudenver.edu \\ ${ }^{3}$ Institut für Mathematik, Universität Wien, Strudlhofgasse 4, A-1090 Wien, \\ Austria \\ Arnold.Neumaier@univie.ac.at
}

\begin{abstract}
A method for incorporating uncertainty in terrain modelling by expressing elevations as fuzzy numbers is proposed. Given a finite set of fuzzy elevations representative of the topographic surface in a certain region, we develop methods to construct surfaces that incorporate the uncertainty. The smoothness and continuity conditions of the surface generating method are maintained. Using this approach, we generalize some classic interpolators and compare them qualitatively. Extensions to wider classes of interpolators follow naturally from our approach. A numerical example is presented to illustrate this idea.
\end{abstract}

\section{Introduction}

Modeling a topographic surface from a finite set of samples is a well-known problem in GIS. Our research interest focuses on the uncertainty of this process. One source of uncertainty in this problem arises from incomplete knowledge of the surface under study, including the uncertainty in the sampled values. Another source is in the choice of the model to describe the phenomena.

The most common method to access spatial uncertainty is to obtain elevation samples from more accurate sources and compare them to Digital Terrain Model (DTM) elevations. Non-spatial statistical parameters like Root Mean Square Error (RMSE) are derived from this method. The RMSE is widely used for many cartography producers like USGS. When the topographic surface is expressed by 
contours, some cartographers use epsilon bands to model elevation uncertainty indirectly ([13]). Another approach to this problem is to apply error propagation techniques to predict uncertainty in DTM from uncertainty in elevation samples [16]. More recently, probability surfaces and simulation techniques have been used [13] and others are based on fuzzy set theory ([1], [17]). We generalize and expand the approach that was developed in [17].

Uncertainty is incorporated in DTM by expressing elevations as fuzzy numbers. A fuzzy number $\tilde{z}$ is a fuzzy set with a special membership function (see [11] or [15]). That distribution is defined by an upper semi-continuous concave membership function $\mu_{z}: \mathbb{R} \rightarrow[0,1]$ with non-empty core. The core is the set of values with membership equal to 1 . When the core has only one element, that element is called the modal value. The interval of positive membership is called support of the fuzzy number (see, for example, [15]). An alternative way to express $\tilde{z}$ is by the $\alpha$ levels, which, in the case of fuzzy numbers, are nested intervals $[z]_{\alpha}$ defined by $\left[z_{\alpha}^{-}, z_{\alpha}^{+}\right]=\{z: \mu(z) \geq \alpha, \alpha \in(0,1]\}$, see figure 1 .

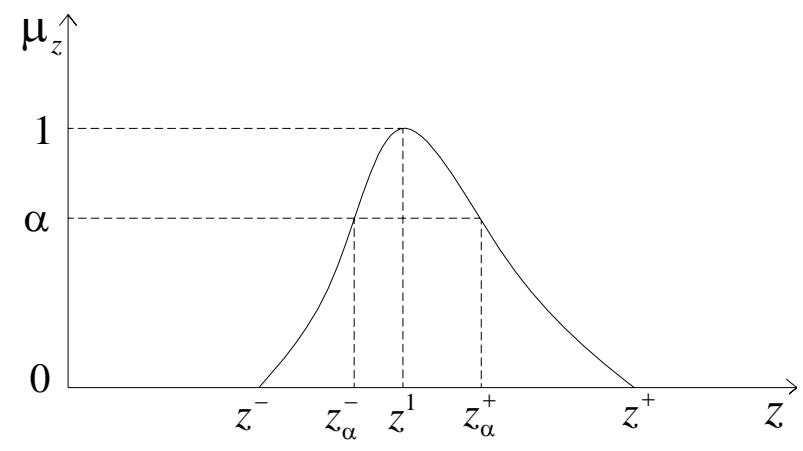

Fig. 1. Fuzzy number with support $\left(z_{\alpha}^{-}, z_{\alpha}^{+}\right)$and modal value $z^{1}$. The $\alpha$-level $[z]_{\alpha}$ is the interval $\left[z_{\alpha}^{-}, z_{\alpha}^{+}\right]$

The problem on which we focus is defined in the following way: given a finite set of fuzzy elevations $\left\{\tilde{z}_{i}=\tilde{z}\left(\mathbf{x}_{i}\right), i=1,2, \ldots, N\right\}$ associated with locations $\left\{\mathbf{x}_{i} \in D \subset \mathbb{R}\right.$ or $\left.\mathbb{R}^{2}, i=1,2, \ldots, N\right\}$, respectively, which are representative of a topographic surface in the region $D$, find a fuzzy-valued function $\tilde{f}(\mathbf{x})$ such that

$$
\tilde{f}(\mathbf{x}) \supseteq \sum_{i=1}^{N} \tilde{z}_{i} \phi_{i}(\mathbf{x}), \forall \mathbf{x} \in D,
$$


where $\phi_{i}$ are basis functions of a class $C$ (Lagrange interpolation polynomials, Hermite polynomials, splines, or radial basis functions). The left side of (1.2), being a fuzzy-valued function, is a fuzzy number for each $\mathbf{x}$. As such it possesses nestedness of the $\alpha$-levels. Moreover smoothness conditions, for example, will be imposed. This is not true of the right side of (1.1) as was proved in [17]. Fuzzy-valued functions used here associate real numbers (position) to fuzzy numbers (elevations).

Uncertainty is incorporated in two ways, via intervals [18] and via fuzzy numbers. While intervals are fuzzy numbers whose core equals its support, it guides our development of fuzzy surface generation, since fuzzy numbers will be viewed as a nested set of $\alpha$-levels and $\alpha$-levels are intervals.

\subsection{Interval case}

We begin by considering the left side of (1.1) as an interval; that is, $f^{I}(\mathbf{x})$ is the particular type of fuzzy number where the membership function is constant in the closed interval and 0 outside. The interpolation condition (1.1) becomes

$$
f^{I}(\mathbf{x}) \supseteq \sum_{i=1}^{N} z_{i}^{I} \phi_{i}(\mathbf{x}), \forall \mathbf{x} \in D \text {, with } z_{i}^{I} \equiv\left[z_{i}^{-}, z_{i}^{+}\right] .
$$

Using the midpoint/radius form ${ }^{1}$ (see [18], [19]) we write

$$
\sum_{i=1}^{N} z_{i}^{I} \phi_{i}(\mathbf{x})=\sum_{i=1}^{N} \breve{z}_{i} \phi_{i}(\mathbf{x})+\sum_{i=1}^{N} \operatorname{rad}\left(z_{i}^{I}\right)\left|\phi_{i}(\mathbf{x})\right|[-1,1]
$$

Note that $\sum_{i=1}^{N} \breve{z}_{i} \phi_{i}(\mathbf{x})$ is the polynomial interpolation at the midpoint $\breve{f}(\mathbf{x})$. The absolute value in (1.3) implies that $\sum_{i=1}^{N} \operatorname{rad}\left(z_{i}^{I}\right)\left|\phi_{i}(\mathbf{x})\right|$ is not in the same class $\mathrm{C}$ of functions from which the basis $\phi_{i}$ are taken (see [17]). Substituting in (1.2) we have

$$
f^{I}(\mathbf{x}) \supseteq \sum_{i=1}^{N} \breve{z}_{i} \phi_{i}(\mathbf{x})+\sum_{i=1}^{N} \operatorname{rad}\left(z_{i}^{I}\right)\left|\phi_{i}(\mathbf{x})\right|[-1,1],
$$

and, because $f^{I}(\mathbf{x})=\breve{f}(\mathbf{x})+\operatorname{rad}\left(f^{I}(\mathbf{x})\right)[-1,1]$, we get the interpolation condition

$$
\operatorname{rad}\left(f^{I}(\mathbf{x})\right) \geq \sum_{i=1}^{N} \operatorname{rad}\left(z_{i}^{I}\right)\left|\phi_{i}(\mathbf{x})\right|, \forall \mathbf{x} \in D .
$$

1 An interval $a^{I}=\left[a^{-}, a^{+}\right]$is defined by its midpoint $\breve{a}=\left(a^{-}+a^{+}\right) / 2$ and radius $\operatorname{rad}\left(a^{I}\right)=\left(a^{+}-a^{-}\right) / 2$ following that $a^{I}=\breve{a}+\operatorname{rad}\left(a^{I}\right)[-1,1]$. 
The consistent representation problem (see [17]) is finding the minimum upper bound of (1.5) such that $f^{-}(\mathbf{x})$ and $f^{+}(\mathbf{x})$ belongs to the same class $C$ of functions as its generator, that is $f^{-}(\mathbf{x})=\sum_{i=1}^{N} \zeta_{i}^{-} \phi_{i}(\mathbf{x})$ and $f^{+}(\mathbf{x})=\sum_{i=1}^{N} \zeta_{i}^{+} \phi_{i}(\mathbf{x})$ belong to class $\mathrm{C}$. Thus, to obtain a consistent representation we solve the semiinfinite optimization problem (see [12])

$$
\begin{aligned}
& \min _{\mathbf{r}}\|\mathbf{r}\| \text {, where } \mathbf{r} \text { is a vector with elements } r_{i}=\operatorname{rad}\left(\zeta_{i}^{I}\right) \\
& \text { subject to } \sum_{i=1}^{N} r_{i} \phi_{i}(\mathbf{x}) \geq \sum_{i=1}^{N} \operatorname{rad}\left(z_{i}^{I}\right)\left|\phi_{i}(\mathbf{x})\right|, \forall \mathbf{x} \in D .
\end{aligned}
$$

\subsection{Fuzzy case}

The interval case can be generalized to the fuzzy case if we express a fuzzy number $\tilde{z}$ by its $\alpha$-levels $[z]_{\alpha}$. Since an $\alpha$-level is an interval $\left[z_{\alpha}^{-}, z_{\alpha}^{+}\right]$(see [14]), we solve the optimization problem (1.6) for every $\alpha$. In the particular case of triangular fuzzy numbers $s^{2} \tilde{z} \equiv\left(z^{-} / z^{1} / z^{+}\right)$, where $\left(z^{-}, z^{+}\right)$is the support and $z^{1}$ the modal value, the problem is solved for all $\alpha$ by applying the interval case to $\left[z^{-}, z^{+}\right]$; the modal value can be treated as a real number. Moreover, trapezoidal fuzzy numbers ${ }^{3}$ can likewise be efficiently treated by applying the interval case to the support $\left(z^{-}, z^{+}\right)$and the core $\left[z_{1}^{-}, z_{1}^{+}\right]$. The complexity of the general case is dependent upon the number of $\alpha$ levels that are required.

\section{Fuzzy Digital Terrain Model (DTM) using semi-infinite programming}

To solve the above optimization problem, we have to deal with an infinite set of constraints. There are algorithms to solve this type of problems (see [12]). In a first approach a grid to discretize the region $D$ was used yielding $K L$ conditions

$$
\sum_{i=1}^{N} \operatorname{rad}\left(\zeta_{i}^{I}\right) \phi_{i}\left(x_{k}, y_{l}\right) \geq \sum_{i=1}^{N} \operatorname{rad}\left(z_{i}^{I}\right)\left|\phi_{i}\left(x_{k}, y_{l}\right)\right|, \forall\left(x_{k}, y_{l}\right) \in D, k=1, \ldots, K, l=1, \ldots, L .
$$

The Optimization Toolbox of Matlab ${ }^{\mathrm{TM}}$ has a minimization function that allows semi-infinite conditions. The prototyping and numerical examples that we present are developed and implemented using this toolbox.

\footnotetext{
${ }^{2}$ A fuzzy number whose membership function has triangular shape.

${ }^{3}$ A fuzzy number whose membership function has trapezoidal shape.
} 


\section{Examples of fuzzy interpolators}

For irregularly distributed points we use a fuzzy linear interpolator based on the Triangulated Irregular Network (TIN) developed in [17]. Fuzzy kriging (see [9]) and thin plate spline (see, for example, [10]) are also well-known interpolators for irregularly distributed data points and can also be generalized to fuzzy interpolation by (1.6).

Fuzzy spline interpolators developed in [17] need a regular grid arrangement of data points. The TIN based interpolator were used to get gridded data from initial irregularly distributed data set. The fuzzy kriging interpolator is based on the variance minimization of the unbiased estimator $\tilde{Z}(\mathbf{x})=\sum_{i} \lambda_{i} \tilde{Z}\left(\mathbf{x}_{i}\right)$, where the weights $\lambda_{i}$ depend on the Gaussian variogram model used to express spatial dependence between sampled values (see [7]).

\section{Numerical Example}

In the example below, elevations are expressed by triangular fuzzy numbers. The initial data points are represented in figure 1 . In figure 2 is a $20 \times 20$ fuzzy grid used to evaluate the fuzzy splines. The resulting fuzzy surfaces are evaluated in a $120 \times 120$ grid and shown in figures 3, 4, 5 and 6, where the middle surface corresponds to modal interpolator values and the fuzzy support is represented by lower and upper surfaces (see figure 9 for detail).

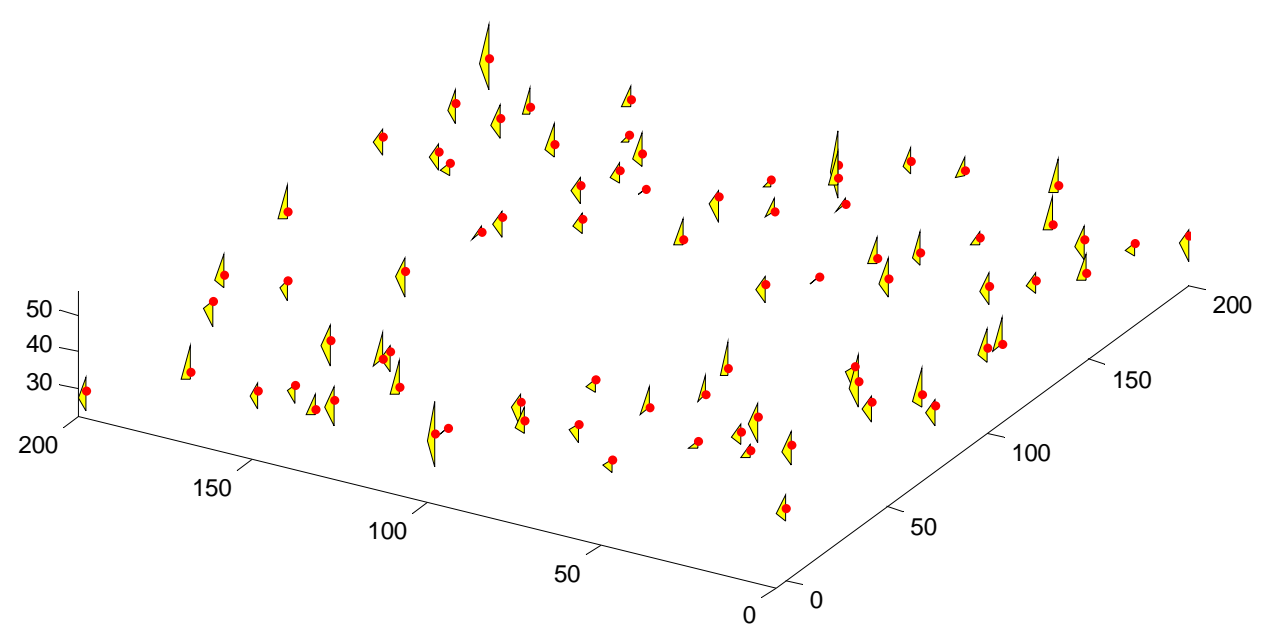

Fig. 2. Fuzzy data (the dots represent modal values) 


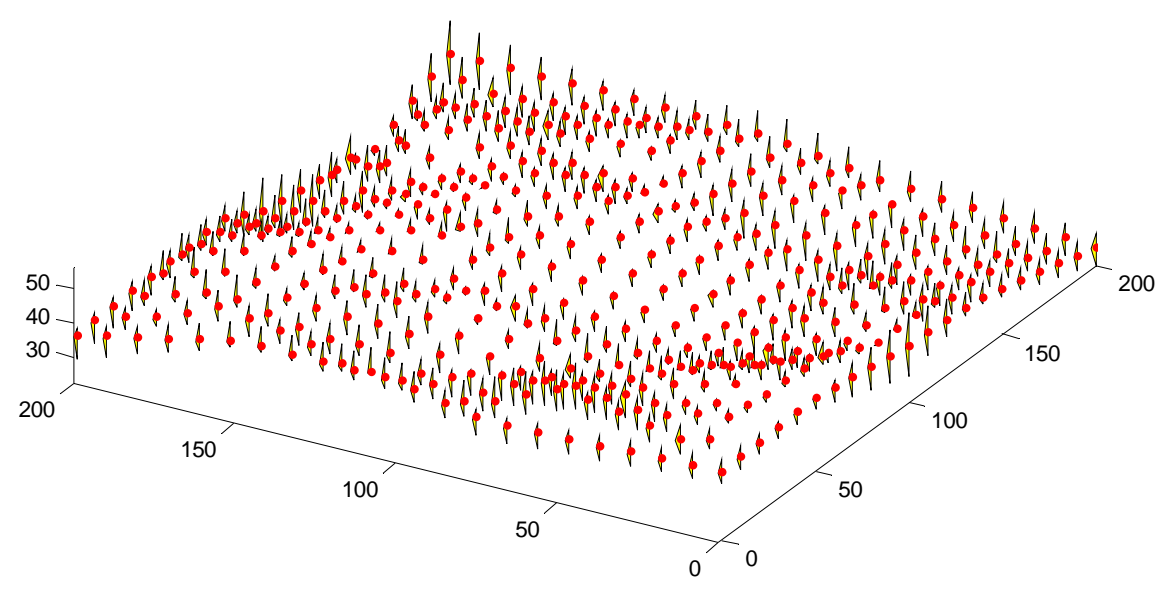

Fig. 3. Gridded fuzzy data

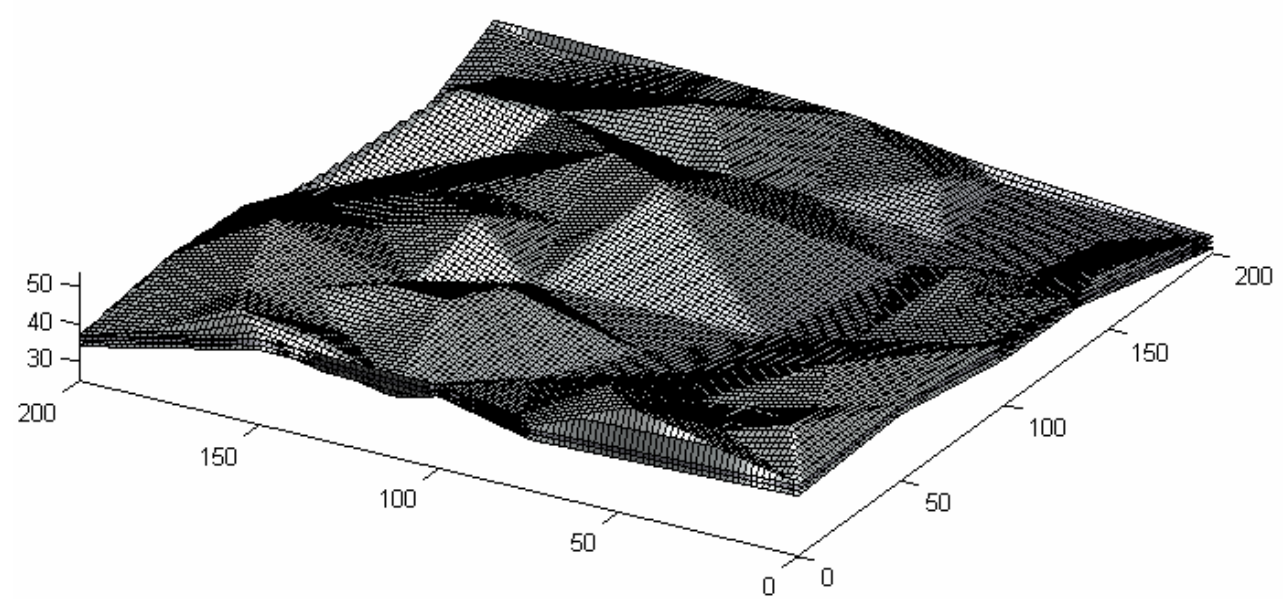

Fig. 4. Fuzzy TIN 


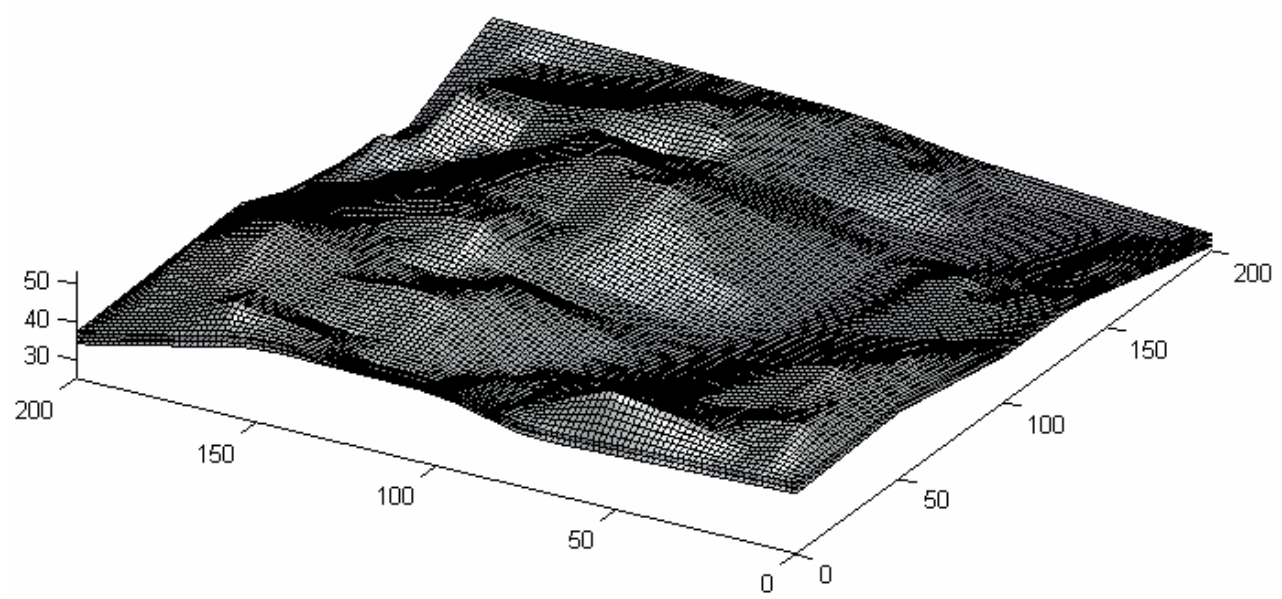

Fig. 5. Fuzzy linear spline

\section{Discussion}

The properties of fuzzy interpolators are similar to the crisp ${ }^{4}$ ones. As it would be expected, the fuzzy linear spline is smoother than the fuzzy polyhedral TIN surface but not so much as the fuzzy cubic spline. The smoothness of fuzzy kriging surface depends on the adopted spatial dependence model. In this case it resembles the TIN surface.

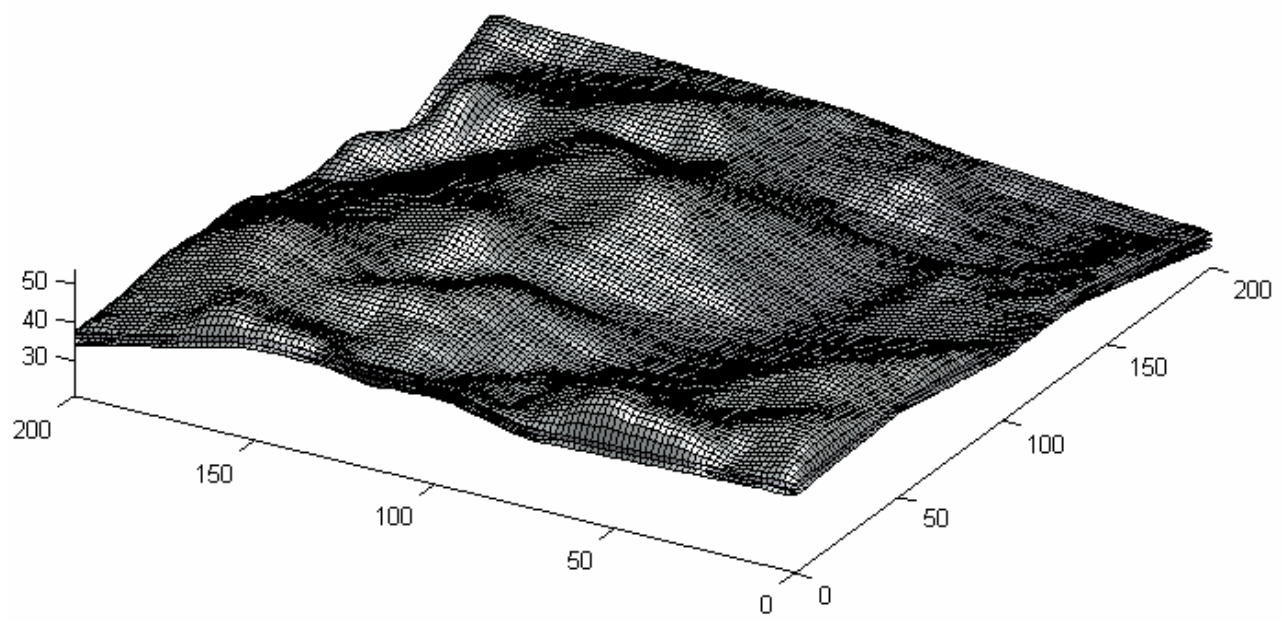

${ }^{4}$ To distinguish between fuzzy entities and classical (no fuzzy) ones, we refer to the latter as crisp 
Fig. 6. Fuzzy cubic spline

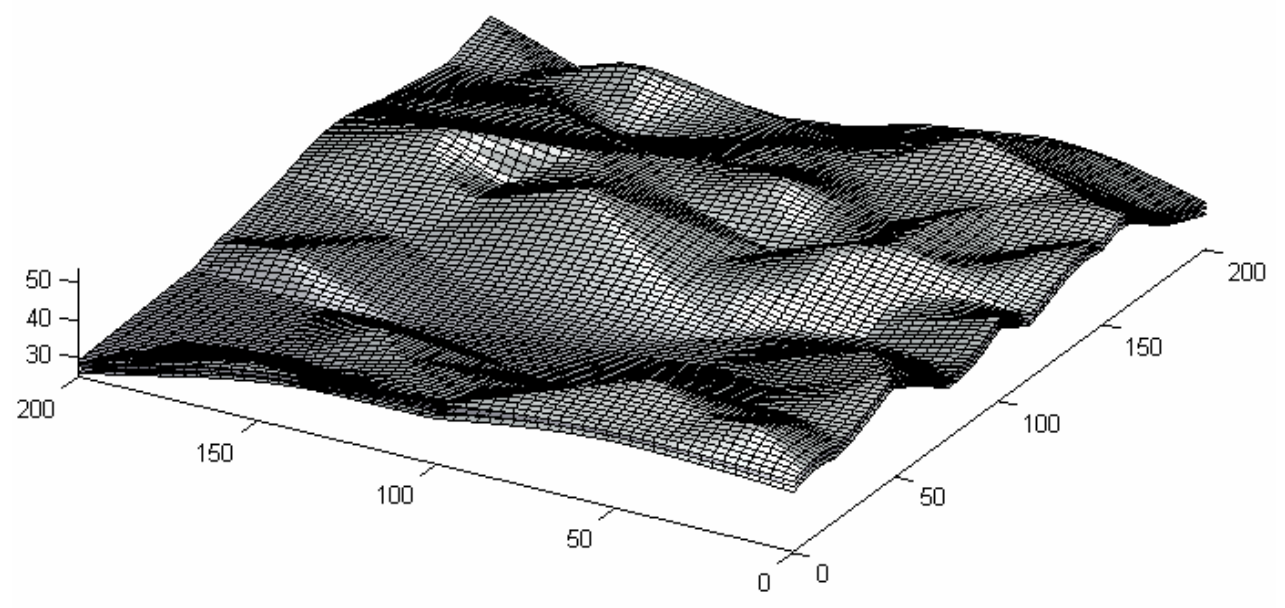

Fig. 7. Fuzzy kriging surface

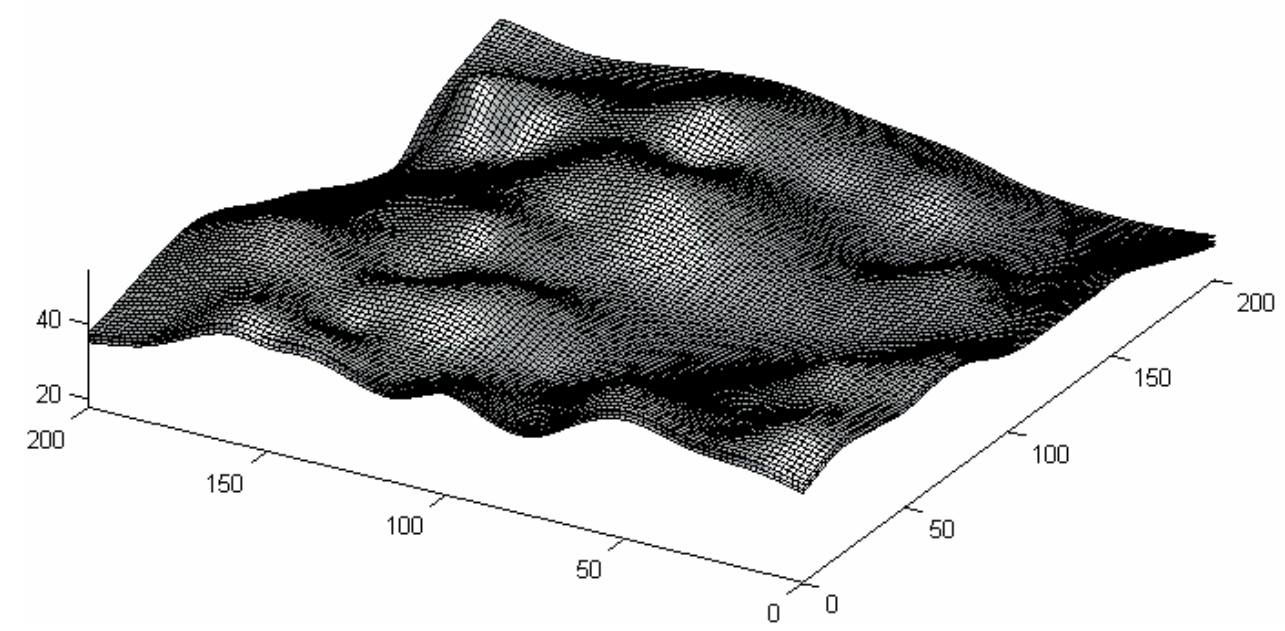

Fig. 8. Fuzzy thin plate spline 


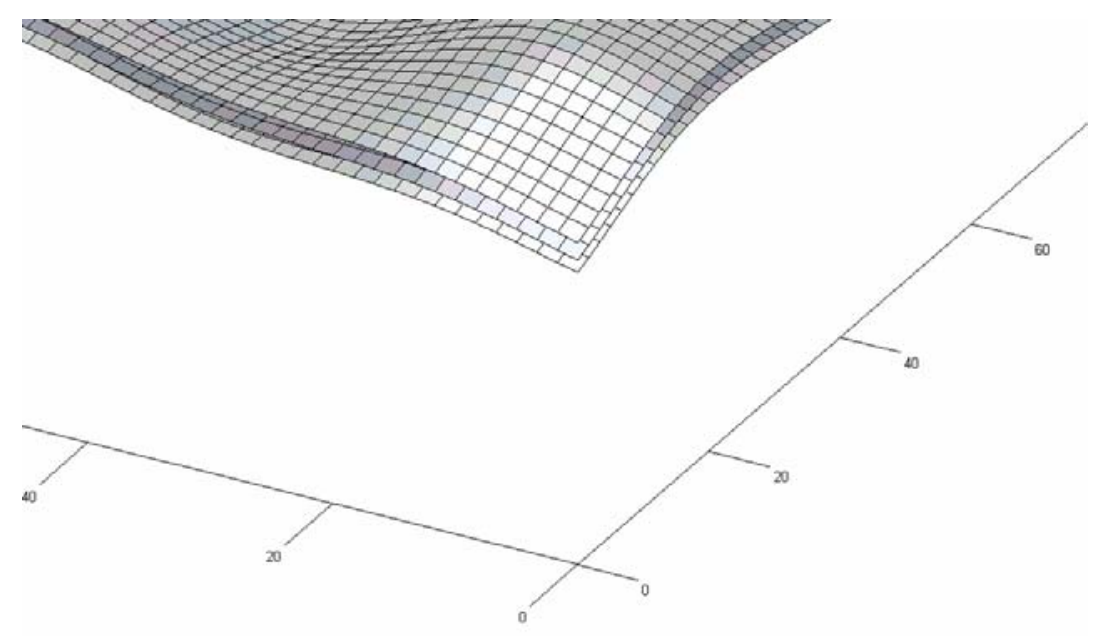

Fig. 9. Detail of fuzzy thin plate spline

The fuzzy thin plate spline is smoother than the other interpolators. Thus, these interpolators can generate surfaces quite different for the same data set. The model choice is one source of uncertainty. One way to include model choice in membership values of fuzzy elevations is to make the different generated surfaces a part of the uncertainty itself. The different elevations given by different interpolators in a same position can be included in a fuzzy elevation for that position. The type of interpolator to choose obviously depends on the characteristics of the surface to be modeled. Another approach would be to take weighted combinations of the various generated surfaces.

The methods we have developed for fuzzy surfaces are able to generate fuzzy DTM's that can be used to study the effect of DTM uncertainty in spatial analyses, like slope and aspect calculations ([20]) or other spatial variables, such as the mapping of the bottom of the ocean ([6], [17]) or visibility ([2]). The proposed methods we have developed can generalize crisp interpolators that are in the form $f(\mathbf{x})=\sum_{i=1}^{N} z_{i} \phi_{i}(\mathbf{x})$ to fuzzy interpolators.

\section{References}

1. Anile, A. M. et al.: Modeling Uncertain Data with Fuzzy B-splines. Fuzzy Sets and Systems 113 (2000) 397-410

2. Anile, A. M. et al.: A Fuzzy Approach to Visibility Maps Creation over Digital Terrains. (to appear in Fuzzy Sets and Systems) 
3. Bardossy, A. et al.: Geostatistics Utilizing Imprecise (Fuzzy) Information. Fuzzy Sets and Systems 31 (1989) 311-328

4. Bardossy, A. et al.: Kriging with Imprecise (Fuzzy) Variograms - I: Theory. Mathematical Geology 22 (1990) 63-79

5. Bardossy, A. et al.: Kriging with Imprecise (Fuzzy) Variograms - II: Application. Mathematical Geology 22 (1990) 81-94

6. Clematis, A. et al.: Parallel Fuzzy Arithmetic for Sea Floor Modeling. Science \& Supercomputing at CINECA. M. Voli Ed. (1998) 539-548

7. Cressie, N.: Statistics for Spatial Data. John Wiley \& Sons, (1993)

8. Deutsch, C. and Journel, A.: GSLIB Geostatistical Software Library and User's Guide. Oxford University Press (1998)

9. Diamond, P.: Fuzzy Kriging. Fuzzy Sets and Systems, (1989) 315-332

10. Dierckx, P.: Curve and Surface Fitting with Splines. Oxford Science Publications, New York (1993)

11. Dubois, D. and Prade, H.: Possibility Theory, an Approach to Computerized Processing of Uncertainty. Plenum Press, New York (1988)

12. Glashoff, K. and Gustafson: Linear Optimization and Approximation, An Introduction to the Theoretical Analysis and Numerical Treatment of Semiinfinite Programs. Springer-Verlag, New York (1983).

13. Hunter, G. J. and Goodchild, M. F.: Dealing with Error in Spatial Databases: A Simple Case Study. Photogrammetric Engineering \& Remote Sensing 61:5 (1995) 529-537

14. Kaufmann, A. and Gupta, M. M.: Introduction to Fuzzy Arithmetic, Theory and Applications. Van Nostrand Reinhold, New York (1991)

15. Klir, G. J. and Yuan, B.: Fuzzy Sets and Fuzzy Logic, Theory and Applications. Prentice Hall Inc., New Jersey (1995)

16. Li, Z.: Mathematical Models of the Accuracy of Digital Terrain Model Surfaces Linearly Constructed from Square Gridded Data. Photogrammetry Record 14:82 (1993) 661-674

17. Lodwick, W. A. and Santos, J.: Constructing Consistent Fuzzy Surfaces from Fuzzy Data. University of Colorado at Denver, Center of Computational Mathematics UCD/CCM Report No.175 (2001) (to appear in Fuzzy Sets and Systems)

18. Moore, R. E.: Methods and Applications of Interval Analysis. SIAM, Philadelphia (1979)

19. Neumaier, A.: Interval Methods for Systems of Equations. Cambridge University Press (1990)

20. Schneider, B.: Uncertainty Propagation in Digital Terrain Modeling with Taylor Methods and Interval Arithmetic. Proceedings Accuracy 2000 (2000) 561-568 